\title{
A novel prodrug strategy to improve the oral absorption of O-desmethylvenlafaxine
}

\author{
MINGYUAN LIU ${ }^{1,2^{*}}$, YANTONG SUN ${ }^{1,3^{*}}$, SEN ZHAO $^{1}$, YOUXIN LI $^{1}$, \\ RIYANG PIAO ${ }^{4}$, YAN YANG ${ }^{5,6}$ and JINGKAI GU ${ }^{1}$ \\ ${ }^{1}$ Research Center for Drug Metabolism, College of Life Science, Jilin University, Changchun, Jilin 130021; \\ ${ }^{2}$ Department of Pharmacology, College of Basic Medical Sciences, Jiamusi University, Jiamusi, Heilongjiang 154007; \\ ${ }^{3}$ School of Pharmaceutical Sciences, Jilin University, Changchun, Jilin 130021; ${ }^{4}$ Jilin Institute of Pharmaceutical Research; \\ ${ }^{5}$ National Engineering Laboratory for AIDS Vaccine, College of Life Science, Jilin University, Changchun, Jilin 130021; \\ ${ }^{6}$ Key Laboratory for Molecular Enzymology and Engineering, The Ministry of Education, \\ School of Life Sciences, Jilin University, Changchun, Jilin 130021, P.R. China
}

Received March 7,2015; Accepted April 11, 2016

DOI: $10.3892 /$ etm.2016.3453

\begin{abstract}
O-Desmethylvenlafaxine (desvenlafaxine, ODV) is the active metabolite of venlafaxine, with similar activity and less risk for pharmacokinetic drug interactions compared to its parent compound venlafaxine. The purpose of this study was to design a series of esters of ODV and assess their potential as ODV prodrugs with improved bioavailability and brain uptake. Seven esters were synthesized and pharmacokinetic screening was performed in rats. The monoester formed on the phenolic hydroxyl of ODV (ODVP-1, ODVP-2, ODVP-3 and ODVP-5) could be degraded to ODV in rat plasma. These four compounds confirmed as possible prodrugs were then studied to evaluated the relative bioavailability of ODV they produced in beagle dogs. ODVP-1, ODVP-2 and ODVP-3 demonstrated higher relative bioavailability of ODV. Finally, ODVP-1, ODVP-2 and ODVP-3 were studied to evaluate their brain uptake in rats. The concentration of ODV in the rat plasma, brain and hypothalamus after administration of ODVP-1, ODVP-2 or ODVP-3 was higher compared with that of ODV. The higher bioavailability, improved pharmacokineics properties and more rapid penetration and translation of
\end{abstract}

Correspondence to: Professor Jingkai Gu, Research Center for Drug Metabolism, College of Life Science, Jilin University, 2699 Qianjin Street, Changchun, Jilin 130021, P.R. China

E-mail: gujk@jlu.edu.cn

Ms. Yan Yang, National Engineering Laboratory for AIDS Vaccine, College of Life Science, Jilin University, 2699 Qianjin Street, Changchun, Jilin 130021, P.R. China

E-mail: yyan@jlu.edu.cn

*Contributed equally

Key words: pharmacokinetics, O-desmethylvenlafaxine, prodrug, bioavailability, rat, dog
ODV suggest that ODVP-1, ODVP-2 or ODVP-3 may warrant further development and application as ODV prodrugs.

\section{Introduction}

Depression is a recurring and life-threatening mental illness with a significant incidence in the population, and represents a major social and economic burden (1). Although several classes of antidepressant medications are currently prescribed to treat depression, serious drawbacks exist that require improvement, such as limitations in efficacy, multiple unwanted side effects, and slow onset of the therapeutic response $(2,3)$. Development of new antidepressants is thus an urgent requirement.

Venlafaxine is an antidepressant of the serotonin-norepinephrine reuptake inhibitor (SNRI) class of drugs that is prescribed for the treatment of major depressive disorder, generalized anxiety disorder and comorbid indications in certain anxiety disorders with depression (4). Venlafaxine is well absorbed in humans and subject to extensive first-pass metabolism in the liver by cytochrome P450 2D6 (CYP2D6) (5). Thus, venlafaxine is susceptible to CYP2D6 polymorphism and the associated variability in pharmacokinetics, efficacy and/or tolerability (6-8). O-desmethylvenlafaxine (ODV), also known as desvenlafaxine succinate, is a synthetic form of the major active metabolite of venlafaxine with antidepressant activity similar to that of venlafaxine but with a longer half-life $(9,10)$. Compared with venlafaxine, direct intake of ODV for the treatment of diseases of the central nervous system has the advantages of being a single compound that is conducive to dosing adjustments and reducing the risk of interactions with other drugs (11-15). However, ODV contains a more exposed hydroxy group compared with venlafaxine, and therefore it has increased hydrophilicity, resulting in lower oral bioavailability. Accordingly, the bioavailability of ODV was confirmed to be $<40 \%$ in beagle dogs (4). An important consequence of low bioavailability is that direct side effects of the unabsorbed drug in the system may be higher. Thus, it is crucial to improve the absorption and bioavailability of ODV. 
Prodrugs are usually designed to improve passive and/or transporter-mediated intestinal absorption to increase oral bioavailability (16-19) and/or tissue-selective delivery (20). Between species differences of hydrolase activity in small intestine and liver result in different exposures to prodrug and active form, and hence different pharmacological effects and toxicities after administration of prodrugs in different animal species, including humans $(21,22)$. The ODV molecule contains two hydroxyls: A phenolic hydroxyl and an alcoholic hydroxyl. In an attempt to increase the bioavailability of ODV in the present study, different vectors were used to bond to a phenolic hydroxyl or two hydroxyls, then seven ODV ester derivatives were successfully synthesized. The purpose of this study was to determine which chemical structure, monoester formed on the phenolic hydroxyl of ODV and/or the diester formed both on the alcoholic hydroxyl and the phenolic hydroxyl of ODV, could be degraded to ODV in the blood. In addition, we aimed to identify ester derivatives with higher bioavailability, improved pharmacokinetics properties and more rapid penetration to further research. Furthermore, in order to evaluate the species differences in bioavailability of ODVP-1, ODVP-2 and ODVP-3 between rats and beagles, although these were evaluated in rats in our previous work (compounds If, Ij and Ik, respectively) $(4,23)$, we assessed the pharmacokinetic profiles of ODVP-1, ODVP-2 and ODVP-3 in beagles.

To these ends, the present study employed in vivo pharmacokinetic analysis of ODV in beagle dogs and pharmacokinetic screening and brain uptake studies in rats. The results indicated that ODVP-1, ODVP-2 and ODVP-3 demonstrated higher bioavailability, improved pharmacokinetics properties, more rapid penetration of ODV, highlighting them as potential development prospects.

\section{Materials and methods}

Animals. Adult male Wistar rats (weight, $250 \pm 20 \mathrm{~g}$ ) were purchased from the Experimental Animal Center of Jilin University (Jilin, China). Adult male beagle dogs (weight, $12 \pm 1 \mathrm{~kg}$ ) were provided by Tianyao Pharmaceutical Co., Ltd. (Tianjin, China) All animals were housed individually at $22 \pm 2^{\circ} \mathrm{C}$ and a relative humidity of $50 \pm 10 \%$ with a 12 -h light/dark cycle, with free access to food and water. The present study was approved by the Ethical Committee of Jilin University. Rats were sacrificed via an overdose of sodium pentobarbital (150 $\mathrm{mg} / \mathrm{kg}$ ) by intraperitoneal injection.

Reagents and instruments. ODV succinate monohydrate, ODVP-1 hydrochloride, ODVP-2 hydrochloride, ODVP-3 succinate, ODVP-4 hydrochloride, ODVP-5 hydrochloride, ODVP-6 hydrochloride and ODVP-7 hydrochloride (derivative content, $>98 \%$ ) were provided by Jilin Institute of Pharmaceutical Research (Jilin, China). The chemical structures of these compounds are shown in Fig. 1. Ethyl ether and dichloromethane were purchased from the Jiayu Chemical Co., Ltd., (Tianjin, China) and Beijing Chemical Factory (Beijing, China), respectively. An Agilent 1100 high performance liquid chromatography (HPLC) system (Agilent Technologies, Inc., Santa Clara, CA, USA) and QTRAP type Triple Quadrupole mass spectrometer (Applied Biosystems; Thermo Fisher Scientific, Inc., Foster City, CA, USA) were used for analysis. LD5-2A

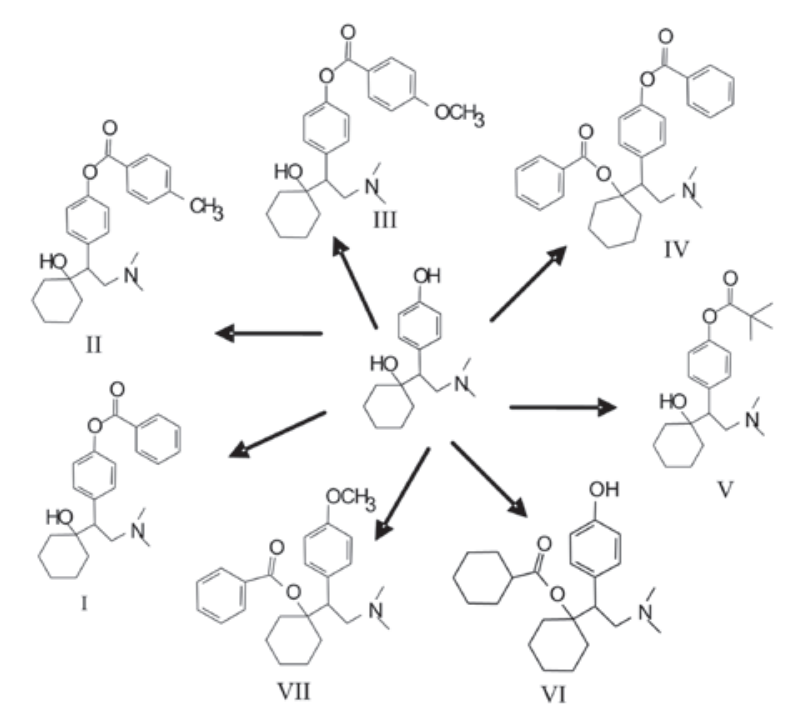

Figure 1. Chemical structures of O-desmethylvenlafaxine (ODV) and its ester derivatives. I: ODVP-1; II: ODVP-2; III: ODVP-3; IV: ODVP-4; V: ODVP-5; VI: ODVP-6; VII: ODVP-7.

centrifuge (Beijing Medical Centrifuge Factory, Beijing, China) and test tubes (Nantong Haizhixing Experimental Co., Ltd., Nantong, China) were used for sample collection.

Administration regimen for the pharmacokinetic screening of the ODV ester derivatives in rats. The seven ODV ester derivatives were prepared in normal saline for oral administration. A total of 21 healthy male Wistar rats were randomly allocated into seven groups ( $\mathrm{n}=3$ per group). After $12 \mathrm{~h}$ of fasting, with free access to water, a dose of $0.043 \mathrm{mmol} / \mathrm{kg}$ ODVP-1, ODVP-2, ODVP-3, ODVP-4, ODVP-5, ODVP-6 or ODVP-7 in $0.5 \mathrm{ml}$ normal saline was intragastrically administered to each of the seven groups. Blood samples $(1.0 \mathrm{ml})$ were extracted from the retrobulbar venous plexus at 5, 10, 30 and $1 \mathrm{~h}$ after administration. The samples were centrifuged in heparinized and dichlorvos-treated test tubes (terminal concentration of dichlorvos, $200 \mu \mathrm{g} / \mathrm{ml})$ for $10 \mathrm{~min}$ at $4^{\circ} \mathrm{C}(1,570 \mathrm{xg})$. The plasma samples were then preserved at $-80^{\circ} \mathrm{C}$ for further analysis.

Chromatography and mass spectrometry for the pharmacokinetic screening of the ODV ester derivatives in rats. The chromatographic column was a ZORBAX Eclipse XDB $\mathrm{C}_{8}$ column (I.D., 4.6x150 mm; particle diameter, $5 \mu \mathrm{m}$; Agilent Technologies, Inc.) and the mobile phase was methanol: $10 \mathrm{mM}$ ammonium acetate $(85: 15, \mathrm{v} / \mathrm{v})$. The flow rate was $1.0 \mathrm{ml} / \mathrm{min}$, the injection volume was $10 \mu \mathrm{l}$ and the column temperature was set at $20^{\circ} \mathrm{C}$. The samples were determined with positive ion mode, the scanned mode was multiple reaction monitoring and the ion reaction used for ODV qualitative analysis was $m / z$ $264.3 \rightarrow m / z$. 58.0.

Pretreatment of plasma samples and HPLC-MS/MS determination for the pharmacokinetic screening of the ODV ester derivatives in rats. Plasma samples $(0.5 \mathrm{ml})$ were added into a Sep-Pak C18 solid phase extraction column (Waters Corporation, Milford, MA, USA) and activated by methanol and water at a pass speed of 30 drops $/ \mathrm{min}$. The samples were washed with $2 \mathrm{ml}$ water and eluted with $2 \mathrm{ml}$ methanol. The collected eluate 
A
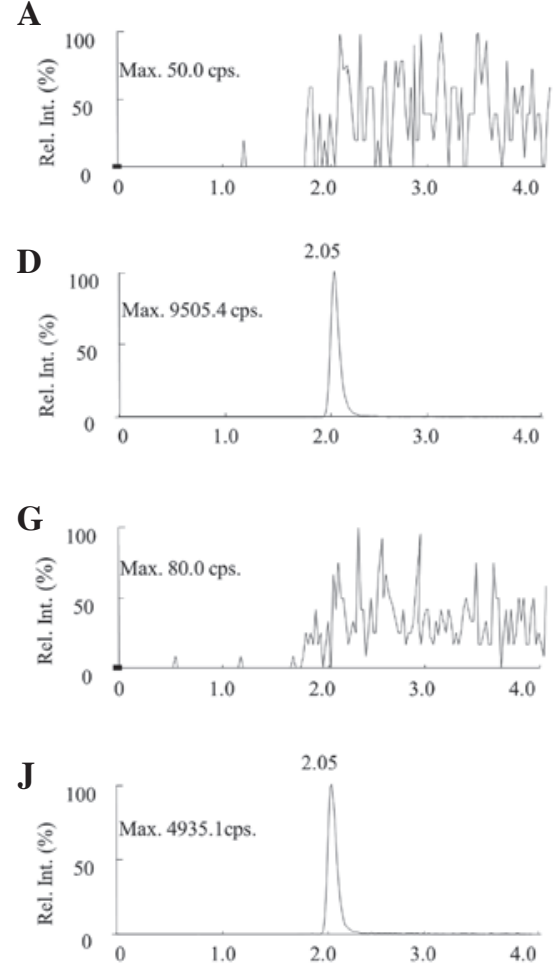
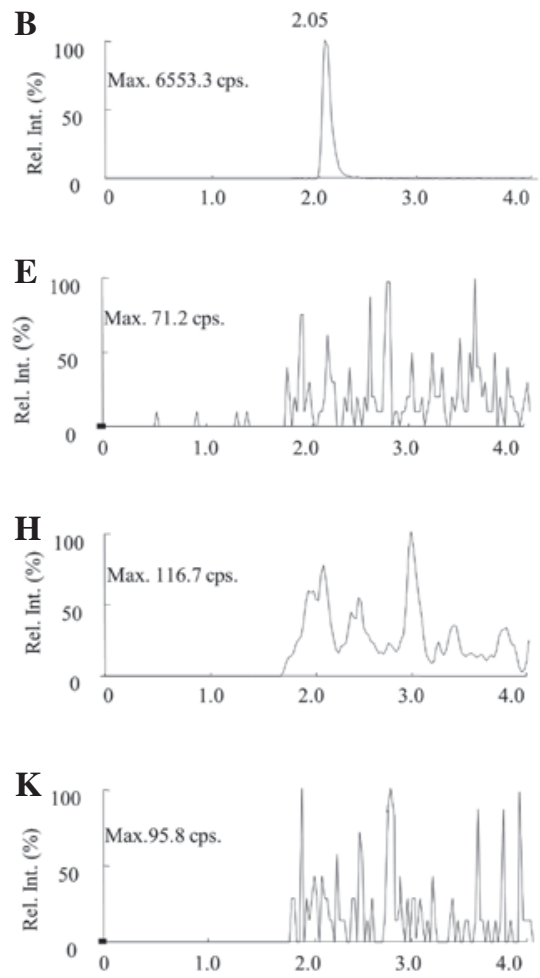
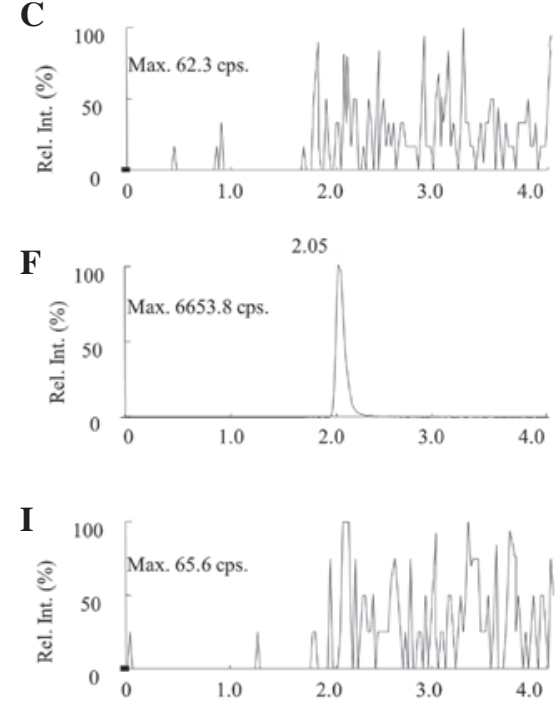

$\mathbf{L}$

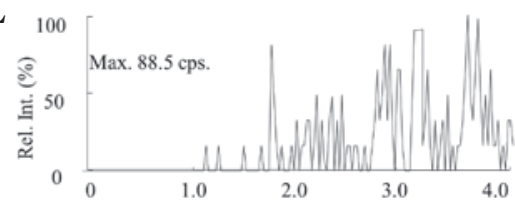

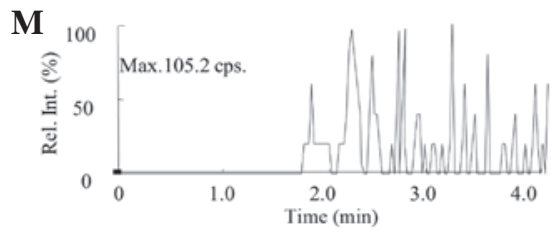

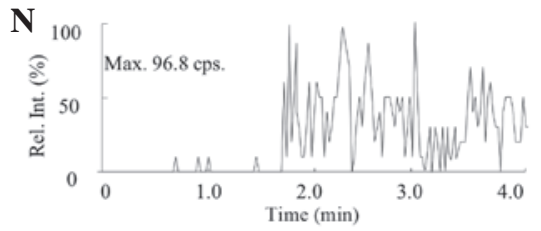

Figure 2. Typical multiple-reaction monitoring chromatograms of the seven ester derivatives of O-desmethylvenlafaxine (ODV) in rat blood (0.043 mmol/kg). Chromatograms of plasma samples of rats prior to and $1 \mathrm{~h}$ following the administration of (A and B) ODVP-1, (C and D) ODVP-2, (E and F) ODVP-3, (G and H) ODVP-4, (I and J) ODVP-5, (K and L) ODVP-6 and (M and N) ODVP-7.

was concentrated to $200 \mu \mathrm{l}$ using a gentle stream of $\mathrm{N}_{2}$, and $10 \mu \mathrm{l}$ was collected for HPLC-MS/MS analysis.

Administration regimen for the pharmacokinetic study of prodrugs in beagle dogs. Pharmacokinetic evaluation was performed in beagle dogs. The test animals were randomly divided into five groups $(n=4)$. Food and water were freely available during the entire experimental period. After a $12 \mathrm{~h}$ fast, each group was administered a single dose of $0.013 \mathrm{mmol} / \mathrm{kg}$ ODV, ODVP-1, ODVP-2, ODVP-3 or ODVP-5.

Sample collection for the pharmacokinetic study of prodrugs in beagle dogs. Blood samples $(1.0 \mathrm{ml})$ were extracted from the small saphenous vein of the legs of beagle dogs prior to and at and $0.083,0.167,0.25,0.5,0.75,1,2,3,4,8,12$ and $24 \mathrm{~h}$ after administration. Blood samples were centrifuged in heparinized and dichlorvos-treated test tubes (terminal concentration of dichlorvos, $200 \mu \mathrm{g} / \mathrm{ml})$ for $10 \mathrm{~min}$ at $4^{\circ} \mathrm{C}(1,570 \mathrm{x} \mathrm{g})$. Plasma samples were stored in a refrigerator protected from light at $-80^{\circ} \mathrm{C}$ for future analysis.

Plasma sample treatment for the pharmacokinetic study of prodrugs in beagle dogs. Plasma samples (100 $\mu \mathrm{l})$ were placed in a test tube with a stopper, and $100 \mu \mathrm{l}$ internal standard solution and $100 \mu \mathrm{l}$ sodium carbonate $(0.1 \mathrm{M})$ were added to the test tube and allowed to mix. A total of $3 \mathrm{ml}$ ethyl ether-dichloromethane $(60: 40, \mathrm{v} / \mathrm{v})$ was then added prior to $10 \mathrm{~min}$ of eddy-mixing, followed by shaking for $10 \mathrm{~min}$ (240 times/min). The samples were then centrifuged for $5 \mathrm{~min}$ at 2,136 $\mathrm{x}$. The upper organic phase was placed in a new tube, and after blow-drying with $\mathrm{N}_{2}$ stream at $25^{\circ} \mathrm{C}, 200 \mu \mathrm{l}$ mobile phase was added to the residue for dissolution. After a second round of eddy-mixing, $10 \mu \mathrm{l}$ of the samples were collected for LC/MS/MS analysis.

Chromatography and mass spectrometry for the pharmacokinetic study of prodrugs in beagle dogs. The chromatographic conditions and mass spectrometer conditions are the same as described above in the "Chromatography and mass spectrometry for the pharmacokinetic screening of the ODV ester derivatives in rats' section. The ion reaction used for qualitative analysis was $m / z, 264.1 \rightarrow m / z, 107.0($ ODV) and $m / z, 256.3 \rightarrow m / z$ 167.1 (internal standard, benzhydramine).

Determination of the concentration of ODVP-1, ODVP-2, $O D V P-3$ and ODV in plasma, brain, and hypothalamus in rats. ODVP-1, ODVP-2, ODVP-3 and ODV were prepared in normal saline for oral administration. Eighty rats were dosed orally with ODVP-1, ODVP-2, ODVP-3 or ODV $(0.043 \mathrm{mmol} / \mathrm{kg}$ in $0.5 \mathrm{ml})$. Food was restricted from $12 \mathrm{~h}$ 
Table I. Pharmacokinetic parameters after intragastric administration of $0.013 \mathrm{mmol} / \mathrm{kg}$ ODV, ODVP-1, ODVP-2, ODVP-3 and ODVP-5 in beagle dogs $(n=4)$, and the relative bioavailability.

\begin{tabular}{lccccc}
\hline Drug & $C_{\max }(\mathrm{ng} / \mathrm{ml})$ & $T_{\max }(\mathrm{h})$ & $t_{1 / 2}(\mathrm{~h})$ & $A U C_{0-t}(\mathrm{ng} \cdot \mathrm{h} / \mathrm{ml})$ & $\mathrm{F}(\%)$ \\
\hline ODV & $100 \pm 40.81$ & $0.5 \pm 0.2$ & $2.07 \pm 0.49$ & $255 \pm 54.30$ & 100 \\
ODVP-1 & $91.5 \pm 12.03$ & $0.9 \pm 0.1$ & $3.27 \pm 1.37$ & $324 \pm 46.25$ & 127 \\
ODVP-2 & $108 \pm 18.57$ & $1.2 \pm 0.6$ & $2.45 \pm 1.08$ & $419 \pm 50.83$ & 164 \\
ODVP-3 & $79.4 \pm 12.32$ & $1.7 \pm 1.0$ & $2.25 \pm 0.22$ & $301 \pm 30.61$ & 118 \\
ODVP-5 & $89.6 \pm 33.29$ & $0.8 \pm 0.2$ & $3.44 \pm 2.48$ & $269 \pm 94.90$ & 105 \\
\hline
\end{tabular}

ODV, O-Desmethylvenlafaxine.

A



C



B

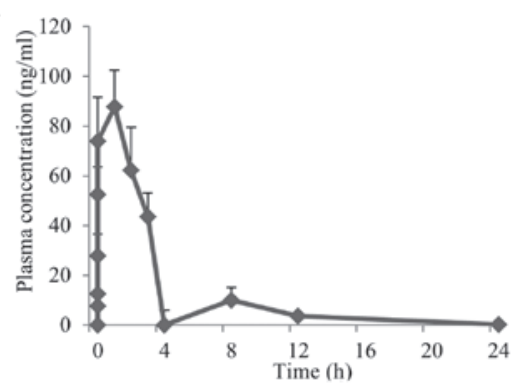

D

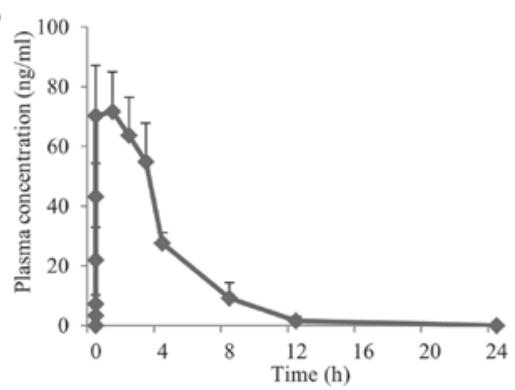

$\mathbf{E}$



Figure 3. O-desmethylvenlafaxine (ODV) average plasma concentration-time curves in plasma from beagle dogs after intragastric administration of $0.013 \mathrm{mmol} / \mathrm{kg}$ ODV, ODVP-1, ODVP-2, ODVP-3 and ODVP-5. (A) ODV; (B) ODVP-1; (C) ODVP-2; (D) ODVP-3; (E) ODVP-5.

predosing to $60 \mathrm{~min}$ postdosing. At $0.25,0.5,1,2$ and $4 \mathrm{~h}$ postdosing, blood samples were drawn from the sinus and collected in heparinized and dichlorvos-treated test tubes (terminal concentration of dichlorvos, $200 \mu \mathrm{g} / \mathrm{ml}$ ) for plasma isolation. At $0.25,0.5,1,2$ and $4 \mathrm{~h}$ postdosing, rats were perfused with $40 \mathrm{ml}$ phosphate-buffered saline $\left(4^{\circ} \mathrm{C}\right)$ via the left ventricle, and the brain was removed and the hypothalamus was dissected. The brain and hypothalamus were placed in ice-cold methanol: water $(50: 50, \mathrm{v} / \mathrm{v})$ in either 2.0 or $1.0 \mathrm{ml}$, respectively, and maintained on ice until tissue homogenization. Plasma and brain tissues were stored at $-80^{\circ} \mathrm{C}$ for further processing. For determination of the concentration of ODVP-1, ODVP-2, ODVP-3 or ODV in tissues, three standard curves (determination of ODVP-1 and ODV, ODVP-2 and ODV, and ODVP-3 and ODV) of plasma or brain tissue $(1.0-1,000 \mathrm{ng} / \mathrm{ml})$ were generated from untreated animals. Samples and standards $(100 \mu \mathrm{l})$ were spiked with $100 \mu \mathrm{l}$ diazepam solution $(1.0 \mu \mathrm{g} / \mathrm{ml})$ in acetonitrile to serve as an internal standard. A total of $3.5 \mathrm{ml}$ N-hexane-dichloromethane-dimethyl carbinol (300:150:15, $\mathrm{v} / \mathrm{v} / \mathrm{v})$ was then added, and $10 \mathrm{~min}$ of eddy-mixing was 

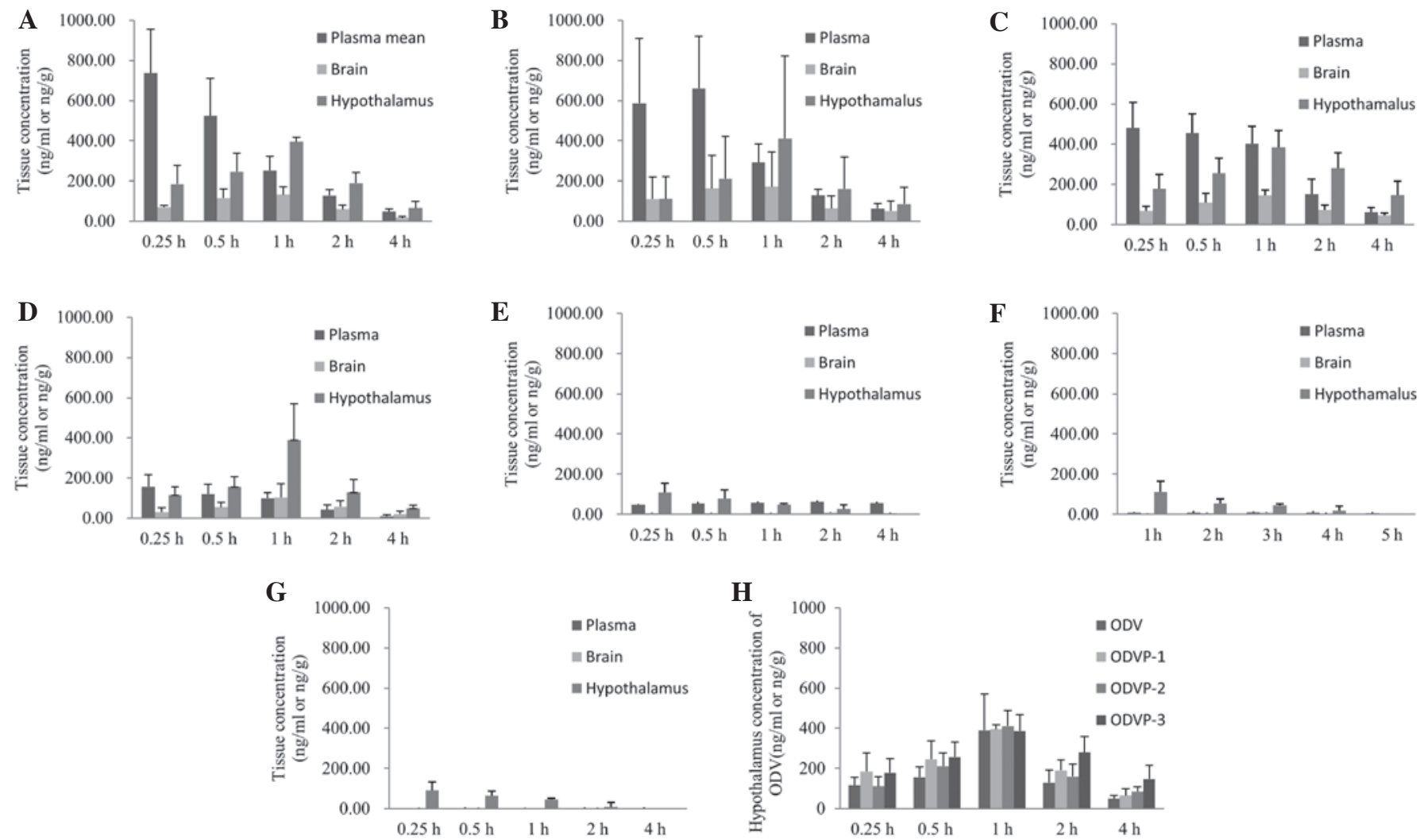

Figure 4. Comparison of the concentrations of ODV in plasma, brain, and hypothalamus after administration of equimolar ODVP-1, ODVP-2, ODVP-3, and ODV $(0.043 \mathrm{mmol} / \mathrm{kg}$, mean $\pm \mathrm{SD}, \mathrm{n}=4)$. ODV in plasma, brain, and hypothalamus after administration of (A) ODVP-1, (B) ODVP-2, (C) ODVP-3 and (D) ODV, respectively; (E) ODVP-1, (F) ODVP-2 and (G) ODVP-3 in the plasma, brain, and hypothalamus after administration of each respective compound. (H) ODV in hypothalamus after administration of ODV, ODVP-1, ODVP-2 and ODVP-3. ODV, O-desmethylvenlafaxine.

performed followed by shaking for $10 \mathrm{~min}$ (240 times/min). The samples were then centrifuged for $5 \mathrm{~min}$ at $2,136 \mathrm{x} \mathrm{g}$. The upper organic phase was placed in a new tube, and after blow-drying with $\mathrm{N}_{2}$ stream at $25^{\circ} \mathrm{C}, 200 \mu$ l methanol: $0.05 \%$ formic acid (50:50, v/v) was added to the residue for dissolution. After a second round of eddy-mixing, $20 \mu 1$ sample was collected for LC/MS/MS analysis.

Statistical analysis. BAPP2.2 software (China Pharmaceutical University, Nanjing, China) was used to calculate the pharmacokinetic parameters. The trapezoidal method was selected for calculation of the $A U C_{0-\mathrm{t}}$ value. Based on the semi-logarithmic map method, the $t_{1} / 2$ was calculated in accordance with the endmost four concentration points of the elimination phase. The $C_{\max }$ and $T_{\max }$ were calculated with the measured values, the $A U C_{0-\mathrm{t}}$ after ODV administration was set as $100 \%$, and the relative bioavailability was calculated according to the $A U C_{0-\mathrm{t}}$ of ODV after the administration of each ODV prodrug.

\section{Results}

Pharmacokinetic screening of the ODV ester derivatives in rats. ODV was clearly detected in the rat plasma of the ODVP-1, ODVP-2, ODVP-3 and ODVP-5 groups at 5, 10, $30 \mathrm{~min}$ and $1 \mathrm{~h}$ after intragastric administration, while ODV was not detected in the rat plasma of the ODVP-4, ODVP-6 and ODVP-7 groups at any time points. The pharmacokinetic screening results showed that the monoesters (ODVP-1,
ODVP-2, ODVP-3 and ODVP-5) formed on the phenolic hydroxyl of ODV could be degraded to ODV in blood, while the diesters (ODVP-4, ODVP-6 and ODVP-7) formed on the alcoholic hydroxyl and the phenolic hydroxy could not be degraded to ODV in blood. The HPLC-MS/MS qualitative detection of ODV in blood samples from each group $1.0 \mathrm{~h}$ after administration is shown in Fig. 2.

Pharmacokinetic study of prodrugs in beagle dogs. ODV, ODVP-1, ODVP-2, ODVP-3 and ODVP-5 (0.013 mmol/ $\mathrm{kg})$ were administered to beagle dogs. The ODV average plasma concentration-time curve for each treatment is shown in Fig. 3. The plasma concentration data was analyzed using the non-compartment model by the BAPP2.2 software. The pharmacokinetic parameters are shown in Table I.

The ODV and the screened ODV prodrugs (ODVP-1, ODVP-2, ODVP-3 and ODVP-5) were orally administered to beagle dogs at a dose of $0.013 \mathrm{mmol} / \mathrm{kg}$. The $A U C_{0-\mathrm{t}}$ values were calculated as 324, 419, 301 and $269 \mathrm{ng} \cdot \mathrm{h} / \mathrm{ml}$, respectively. Taking the $A U C_{0-\mathrm{t}}$ of $255 \mathrm{ng} \cdot \mathrm{h} / \mathrm{ml}$ of ODV as $100 \%$, the relative bioavailabilities of ODVP-1, ODVP-2, ODVP-3 and ODVP-5 were calculated at 127, 164, 118 and $105 \%$, respectively. The bioavailabilities of ODVP-1, ODVP-2, ODVP-3 and ODVP-5 were all increased compared with ODV succinate. Notably, the bioavailability of ODVP-2 was enhanced by $>60 \%$, indicating a marked improvement.

The $C_{\max }$ of ODV was $100 \mathrm{ng} / \mathrm{ml}$, and the $C_{\max }$ values of ODVP-1, ODVP-2, ODVP-3 and ODVP-5 were 91.5, 108, 
79.4 and $89.6 \mathrm{ng} / \mathrm{ml}$, respectively. The $C_{\max }$ of each ODV prodrug was relatively close to the $C_{\max }$ of ODV. While ODVP-3 reached the maximum ODV concentration at $1.7 \mathrm{~h}$, ODVP-1, ODVP-2 and ODVP-5 all reached the maximum ODV concentration at $\sim 1.0 \mathrm{~h}$. Notably, all of their peak times were later compared with the original drug ODV $(0.5 \mathrm{~h})$.

The half-lives of ODV after the administration of ODVP-1, ODVP-2, ODVP-3 and ODVP-5 were 3.27, 2.45, 2.25 and $3.44 \mathrm{~h}$, respectively. All were longer than the half-life of ODV (2.07 h).

Concentrations of ODVP-1, ODVP-2, ODVP-3 and ODV in the plasma, brain and hypothalamus in rats after oral administration. Concentrations of ODVP-1, ODVP-2, ODVP-3 and ODV were determined in plasma, total brain and hypothalamus in rats for each time point over a 4-h period (Fig. 4). Total brain represents the remainder of brain tissue after dissecting the hypothalamus.

The concentration of ODV in plasma, brain and hypothalamus after administration of ODVP-1, ODVP-2 or ODVP-3 was higher compared with that of ODV at the same time point after administration of ODV. Trace quantities of ODVP-1, ODVP-2 or ODVP-3 were detected in the blood and hypothalamus after administration of ODVP-1 over a $2 \mathrm{~h}$ period. We clearly found that ODVP-1, ODVP-2, or ODVP-3 were absorbed into blood and translated into ODV rapidly.

\section{Discussion}

Prodrugs are bioreversible derivates of drug molecules that transform by carboxylesterase in vivo to release the active parent drug (24). The aim of this approach is to increase the usefulness of a drug by improving the physicochemical, biopharmaceutical or pharmacokinetic properties of the compound (25-28). By chemically modifying an active agent, various mitigating factors to efficacy may be overcome, such as poor aqueous solubility, chemical instability, insufficient oral absorption, rapid presystemic metabolism, inadequate brain penetration, toxicity and irritation (25-28). Prodrugs may also prolong the duration of drug action (29). The ODV molecule contains two hydroxyls: A phenolic hydroxyl and an alcoholic hydroxyl. We used chemical bonding to connect a phenolic hydroxyl or two hydroxyls, and different vectors were used to successfully synthesize seven ODV ester derivatives. In order to conduct the pharmacokinetic evaluation of the ODV ester derivatives, equimolar quantities of the newly synthesized ODV ester derivatives were intragastrically administered to Wistar rats. Results showed that among the seven newly synthesized compounds, the monoester formed on the phenolic hydroxyl of ODV (ODVP-1, ODVP-2, ODVP-3 and ODVP-5) could be degraded to ODV in rat plasma, confirming these as possible prodrugs, whilst the diester formed both on the alcoholic hydroxyl and phenolic hydroxyl of ODV (ODVP-4, ODVP 6 and ODVP-7) could not be degraded to generate ODV in rat plasma, eliminating them for consideration as potential prodrugs.

Carboxylesterase is an enzyme that is capable of hydrolyzing a wide variety of carboxylic acid esters (30). The enzyme is widely distributed in nature, being particularly common in the mammalian liver (31-33). Blood from rodents and beagle dogs is routinely treated with dichlorvos to inhibit esterase-catalysed ex vivo hydrolysis of ester derivatives $(34,35)$. In the present study, the efficacy of this inhibitor was demonstrated in pooled samples of rat or dog blood and plasma incubated with newly synthesized ODV ester derivatives. The results of the present study demonstrated that treatment of fresh blood with dichlorvos is effective in preventing the ex vivo decomposition of the ODV ester prodrug.

Species differences of hydrolase activity in small intestine and liver can result in different exposures to prodrug and active form after administration of prodrugs. The relative bioavailability of ODV after administration of ODVP-1, ODVP-2 and ODVP-3 in beagles (127, 164 and 118\%, respectively) were all higher than those of ODV in rats, which were 98.4, 110 and $104 \%$, respectively, as reported in our previous paper (23). This data indicates that the hydrolytic activity in canine small intestine is lower than in rats, so the absorption and bioavailability of ODVP-1, ODVP-2 and ODVP-3 are all higher in canines than in rats. These finding coincide with the study performed by Taketani et al (36) which reported that no hydrolase activity was detected in dog small intestine.

Following the oral administration of ODVP-1, ODVP-2 or ODVP-3, the compounds were absorbed into the blood from the stomach and small intestine. The majority of ODVP-1, ODVP-2 or ODVP-3 in the blood was translated into ODV rapidly, and a trace quantity in the blood was not degraded that rapidly entered the brain. The present study evaluated brain concentrations of ODV, ODVP-1, ODVP-2 or ODVP-3 over time after oral administration in male rats. ODVP-1, ODVP-2 and ODVP-3 demonstrated rapid hypothalamus penetration, with hypothalamus concentrations in excess of those noted in the plasma after a single oral administration of ODVP-1, ODVP-2 or ODVP-3. The results shown in Table I and Fig. 3 indicate that ODVP-1, ODVP-2 and ODVP-3 demonstrate higher bioavailability and improved pharmacokinetics compared with equimolar ODV. In addition, ODV and ODVP-1, ODVP-2 or ODVP-3 were all detected in the brain and hypothalamus after oral administration of ODVP-1, ODVP-2 or ODVP-3. The difference is that ODV has been maintained at relatively high concentrations for $0-4 \mathrm{~h}$, while ODVP-1, ODVP-2 or ODVP-3 last for only $0-2 \mathrm{~h}$ to maintain a certain concentration and significantly decreased with time.

In summary, ODVP-1, ODVP-2, and ODVP-3 demonstrated higher bioavailability and improved pharmacokinetics properties than ODV, penetrated the male rat brain and hypothalamus rapidly. Collectively, the results of this study indicate that these ODV prodrugs may be useful for further development and application.

\section{Acknowledgements}

This research was financially supported by the Natural Science Foundation of Heilongjiang Province (grant no. H201361), Scientific project (grant no. 12511571) of Heilongjiang Provincial Department of Education, the National Natural Science Foundation of China (grant nos. 30973587 and 81102383), the Science and Technology Major Specialized Projects for 'significant new drugs creation' of the 12th five-year plan (grant nos. 2012ZX09303-015 and 2014ZX09303303), the National Key Technology R\&D Program of the Ministry 
of Science and Technology (grant no. 2012BAI30B00) and the China Equipment and Education Resources System (grant no. CERS-1-70).

\section{References}

1. Wong ML and Licinio J: Research and treatment approaches to depression. Nat Rev Neurosci 2: 343-351, 2001.

2. Melfi CA, Chawla AJ, Croghan TW, Hanna MP, Kennedy $\mathrm{S}$ and Sredl K: The effects of adherence to antidepressant treatment guidelines on relapse and recurrence of depression. Arch Gen Psychiatry 55: 1128-1132, 1998.

3. Gumnick JF and Nemeroff CB: Problems with currently available antidepressants. J Clin Psychiatry 61: 5-15, 2000.

4. Tian JW, Jiang WL, Zhong Y, Meng Q, Gai Y, Zhu HB, Hou J Xing Y and Li YX: Preclinical pharmacology of TP1, a nove potent triple reuptake inhibitor with antidepressant properties. Neuroscience 196: 124-130, 2011.

5. Otton SV, Ball SE, Cheung SW, Inaba T, Rudolph RL and Sellers EM: Venlafaxine oxidation in vitro is catalysed by CYP2D6. Br J Clin Pharmacol 41: 149-156, 1996.

6. Klamerus KJ, Maloney K, Rudolph RL, Sisenwine SF, Jusko WJ and Chiang ST: Introduction of a composite parameter to the pharmacokinetics of venlafaxine and its active O-desmethyl metabolite. J Clin Pharmacol 32: 716-724, 1992.

7. Schweizer E, Thielen RJ and Frazer A: Venlafaxine: A novel antidepressant compound. Expert Opin Investig Drugs 6: 65-78, 1997.

8. Perry $R$ and Cassagnol $M$ : Desvenlafaxine: A new serotonin-norepinephrine reuptake inhibitor for the treatment of adults with major depressive disorder. Clin Ther 31 : 1374-1404, 2009.

9. Rudorfer MV and Potter WZ: The role of metabolites of antidepressants in the treatment of depression. CNS Drugs 7: 273-312, 1997.

10. Ereshefsky L and Dugan D: Review of the pharmacokinetics, pharmacogenetics, and drug interaction potential of antidepressants: Focus on venlafaxine. Depress Anxiety 12 (Suppl 1): S30-S44, 2000.

11. Nichols AI, Focht K, Jiang Q, Preskorn SH and Kane CP: Pharmacokinetics of venlafaxine extended release $75 \mathrm{mg}$ and desvenlafaxine $50 \mathrm{mg}$ in healthy CYP2D6 extensive and poor metabolizers: A randomized, open-label, two-period, parallel-group, crossover study. Clin Drug Investig 31: $155-167,2011$

12. Nichols AI, Abell M, Chen Y, Behrle JA, Frick G and Paul J: Effects of desvenlafaxine on the pharmacokinetics of desipramine in healthy adults. Int Clin Psychopharmacol 28: 99-105, 2013.

13. Reddy S, Kane C, Pitrosky B, Musgnung J, Ninan PT and Guico-Pabia CJ: Clinical utility of desvenlafaxine $50 \mathrm{mg} / \mathrm{d}$ for treating MDD: A review of two randomized placebo-controlled trials for the practicing physician. Curr Med Res Opin 26: 139-150, 2010.

14. Sproule BA, Hazra M and Pollock BG: Desvenlafaxine succinate for major depressive disorder. Drugs Today (Barc) 44: 475-487, 2008.

15. Nichols AI, Tourian KA, Tse SY and Paul J: Desvenlafaxine for major depressive disorder: Incremental clinical benefits from a second-generation serotonin-norepinephrine reuptake inhibitor. Expert Opin Drug Metab Toxicol 6: 1565-1574, 2010.

16. Mizuno N, Niwa T, Yotsumoto Y and Sugiyama Y: Impact of drug transporter studies on drug discovery and development. Pharmacol Rev 55: 425-461, 2003.
17. Baudy RB, Butera JA, Abou-Gharbia MA, Chen H, Harrison B, Jain U, Magolda R, Sze JY, Brandt MR, Cummons TA, et al: Prodrugs of perzinfotel with improved oral bioavailability. J Med Chem 52: 771-778, 2009.

18. Xie Q, Wang X, Jiang Z and Qiu Z: Design, synthesis, and bioavailability evaluation of coumarin-based prodrug of meptazinol. Bioorg Med Chem Lett 15: 4953-4956, 2005.

19. Kahns AH, Møss J and Bundgaard H: Improved oral bioavailability of salicylamide in rabbits by a 1,3-benzoxazine-2,4-dione prodrug. Int J Pharm 78: 199-202, 1992.

20. Horn AS, Kelly P, Westerink BH and Dijkstra DA: A prodrug of ADTN: Selectivity of dopaminergic action and brain levels of ADTN. Eur J Pharmacol 60: 95-99, 1979.

21. Ecobichon D: Relative amounts of hepatic and renal carboxylesterase in mammalian species. Res Commun Chem Pathol Pharmacol 3: 629-636, 1972.

22. Cook CS, Karabatsos PJ, Schoenhard Gl and Karim A: Species dependent esterase activities for hydrolysis of an anti-HIV prodrug glycovir and bioavailability of active SC-48334. Pharm Res 12: 1158-64, 1995

23. Zhang Y, Yang Y, Zhao S, Yang Z, Yang H, Fawcett JP, Li Y, $\mathrm{Gu} \mathrm{J}$ and Sun T: Phenolic esters of O-desmethylvenlafaxine with improved oral bioavailability and brain uptake. Molecules 18: 14920-14934, 2013.

24. Liederer BM and Borchardt RT: Enzymes involved in the bioconversion of ester-based prodrugs. J Pharm Sci 95: 1177-1195, 2006.

25. Beaumont K, Webster R, Gardner I and Dack K: Design of ester prodrugs to enhance oral absorption of poorly permeable compounds: challenges to the discovery scientist. Curr Drug Metab 4: 461-485, 2003.

26. Ettmayer P, Amidon GL, Clement B and Testa B: Lessons learned from marketed and investigational prodrugs. J Med Chem 47: 2393-2404, 2004.

27. Testa B: Prodrug research: Futile or fertile? Biochem Pharmacol 68: 2097-2106, 2004.

28. Stella VJ and Nti-Addae KW: Prodrug strategies to overcome poor water solubility. Adv Drug Deliv Rev 59: 677-694, 2007.

29. Rautio J, Kumpulainen H, Heimbach T, Oliyai R, Oh D, Järvinen T and Savolainen J: Prodrugs: Design and clinical applications. Nat Rev Drug Discov 7: 255-270, 2008.

30. Laizure SC1, Herring V, Hu Z, Witbrodt K and Parker RB: The role of human carboxylesterases in drug metabolism: Have we overlooked their importance? Pharmacotherapy 33: 210-222, 2013.

31. Pindel EV, Kedishvili NY, Abraham TL, Brzezinski MR, Zhang J, Dean RA and Bosron WF: Purification and cloning of a broad substrate specificity human liver carboxylesterase that catalyzes the hydrolysis of cocaine and heroin. J Biol Chem 272: 14769-14775, 1997.

32. Schewer H, Langmann T, Daig R, Becker A, Aslandis C and Schmitz G: Molecular cloning and characterization of a novel putative carboxylesterase, present in human intestine and liver. Biochem Biophys Res Commun 233: 117-120, 1997.

33. Holmes RS, Glenn JP, Vandeberg JL and Cox LA: Baboon carboxylesterases 1 and 2: Sequences, structures and phylogenetics relationships with human and other primate carboxylesterases. J Med Primatol 38: 27-38, 2009.

34. Song X and Siahaan TJ: Synthesis and stability study of a modified phenylpropionic acid linker-based esterase-sensitive prodrug. Bioorg Med Chem Lett 12: 3439-3442, 2002.

35. Sakaguchi K, Nagayama M, Masaoka T, Nishimura A, Kageyama K, Shirai M and Akahori F: Effects of fenthion, isoxathion, dichlorvos and propaphos on the serum cholinesterase isoenzyme patterns of dogs. Vet Hum Toxicol 39: 1-5, 1997.

36. Taketani M, Shii M, Ohura K, Ninomiya S and Imai $T$ : Carboxylesterase in the liver and small intestine of experimental animals and human. Life Sci 81: 924-932, 2007. 